\title{
KAM tori in 1D random discrete nonlinear Schrödinger model?
}

\author{
Magnus Johansson ${ }^{1,4}$, Georgios Kopidakis ${ }^{2,4}$ and Serge Aubry ${ }^{3,4}$ \\ 1 Department of Physics, Chemistry and Biology (IFM), Linköping University, SE-581 83 Linköping, Sweden \\ 2 Department of Materials Science and Technology, University of Crete, GR-71003 Heraklion, Greece \\ 3 Laboratoire Léon Brillouin, CEA Saclay, 91191 Gif-sur-Yvette, France \\ 4 Max Planck Institute for the Physics of Complex Systems, Nöthnitzer Str. 38, D-01187 Dresden, Germany
}

PACS 05.45.-a - Nonlinear dynamics and chaos

PACS $45.05 .+\mathrm{x}$ - General theory of classical mechanics of discrete systems

PACS 42.25.Dd - Wave propagation in random media

\begin{abstract}
We suggest that KAM theory could be extended for certain infinite-dimensional systems with purely discrete linear spectrum. We provide empirical arguments for the existence of square summable infinite-dimensional invariant tori in the random discrete nonlinear Schrödinger equation, appearing with a finite probability for a given initial condition with sufficiently small norm. Numerical support for the existence of a fat Cantor set of initial conditions generating almost-periodic oscillations is obtained by analyzing (i) sets of recurrent trajectories over successively larger time scales, and (ii) finite-time Lyapunov exponents. The norm region where such KAM-like tori may exist shrinks to zero when the disorder strength goes to zero and the localization length diverges.
\end{abstract}

KAM theory 1] predicts that, under generic conditions, Hamiltonian systems with a finite number of degrees of freedom, $N$, close enough to an integrable limit exhibit quasiperiodic trajectories which are dense on invariant $N$ dimensional tori in a $2 N$-dimensional phase space. The $N$ fundamental frequencies of those trajectories depend on the torus. When some integer combination of the frequencies vanishes, resonance is obtained, and tori which - are resonant or almost resonant generally break up into chaotic trajectories when the Hamiltonian is perturbed from the integrable limit. Since there are infinitely many possible resonances, infinitely many gaps appear densely in phase space. As the volume of the gaps drops exponentially with the order of the corresponding resonance, the persisting tori form a fat Cantor set (i.e., of nonvanishing Lebesgue measure), which goes to full measure at the integrable limit. There is no general extension of the KAM theory for infinite systems $(N=\infty)$, except for some special models [2,3]. However, it is generally believed that most KAM tori disappear when the dimension of the dynamical system is infinite (see, e.g., 4]).

Simple empirical arguments confirm that KAM tori, which are spatially localized (square summable, $l_{2}$ ) cannot survive when the spectrum of the linearized system is absolutely continuous in some frequency interval. This situation occurs, e.g., for spatially periodic arrays of coupled anharmonic oscillators. In such models, any hypothetical quasiperiodic solution with at least two incommensurate frequencies would generate harmonics densely on the real axis, overlapping with the interval of the absolutely continuous part. Thus, these harmonics would radiate energy towards infinity so that the localized energy could not be conserved [5]. (By contrast, simple periodic solutions may remain localized, forming intrinsic localized modes ["discrete breathers"] which may be dynamical attractors for some initial conditions [6].) However, these arguments do not hold when the linear spectrum is purely discrete, and it is known, e.g., that spatially localized periodic solutions with frequencies inside the linear spectrum exist generically in systems with linear Anderson localization [7,8,

We consider here the one-dimensional random discrete nonlinear Schrödinger (DNLS) system (see, e.g., [9]14]),

$$
i \dot{\psi}_{n}=\left(\epsilon_{n}+\chi\left|\psi_{n}\right|^{2}\right) \psi_{n}-C\left(\psi_{n+1}+\psi_{n-1}\right),
$$

with integrable limits at $\chi=0$ (linear limit) and $C=0$ (anticontinuous limit). The random onsite energies $\epsilon_{n}$ are uniformly distributed in the interval $[-W / 2: W / 2]$. (See [15] for a direct experimental realization of (1) with optical waveguide arrays.) Then the linear spectrum $(\chi=0)$ is discrete and the corresponding eigenstates are $l_{2}$ (exponentially localized). Bourgain and Wang [16] have proven that close enough to the linear limit, eq. (1) 
exhibits quasiperiodic solutions corresponding to finitedimensional tori in phase space. Since in finite systems, invariant tori with dimension $P<N$ generally have zero measure, we expect similarly the solutions found in [16] to have zero probability to occur in an infinite system. In this work, we provide empirical arguments, supported by numerical calculations, suggesting that eq. (1) may also sustain infinite dimensional invariant tori of almost periodic 1 trajectories, which are $l_{2}$ and could be found with nonvanishing probability for an initial condition at small enough norm. Fröhlich, Spencer and Wayne proved the existence of such tori [2], but only in a special family of modified random DNLS-type systems not including eq. (1).

Our empirical arguments can be summarized as follows: We consider an arbitrary $l_{2}$ small-amplitude initial condition, corresponding to a distribution of excited linear modes coupled by weak nonlinearities. Estimating the probability that these couplings induce resonances between the linear modes, we find this probability to vanish in the limit of small norm. Since our argument explicitly uses the fact that the linear spectrum is discrete with exponentially localized eigenstates, it does not hold for a system with an absolutely continuous part in its spectrum. Moreover, we predict that the existence domain of KAMlike tori shrinks to zero in the limit of weak disorder, or equivalently long localization length.

For the linearized eq. (11), the discrete (countable) set of eigenvalues $\omega_{p}$ is associated with a basis of real $l_{2}$ eigenstates $\left\{\phi_{n}^{(p)}\right\}: \omega_{p} \phi_{n}^{(p)}=\epsilon_{n} \phi_{n}^{(p)}-C\left(\phi_{n+1}^{(p)}+\phi_{n-1}^{(p)}\right)$. Expanding $\left\{\psi_{n}\right\}$ in this basis, $\psi_{n}(t)=\sum_{p} \mu_{p}(t) \phi_{n}^{(p)}$, yields $\left|\psi_{n}(t)\right|^{2}=\sum_{p, p^{\prime}} \mu_{p}^{\star}(t) \mu_{p^{\prime}}(t) \phi_{n}^{(p)} \phi_{n}^{\left(p^{\prime}\right)}$, and the norm square

$$
\mathcal{N} \equiv \sum_{n}\left|\psi_{n}\right|^{2}=\sum_{p}\left|\mu_{p}\right|^{2}
$$

which is the second conserved quantity of eq. (10) (in addition to the Hamiltonian). Then, we obtain for the new complex coordinates $\mu_{p}$ (cf, e.g., [9, 13, 14]),

$$
i \dot{\mu}_{p}=\omega_{p} \mu_{p}+\sum_{p^{\prime}} C_{p, p^{\prime}}(t) \mu_{p^{\prime}}(t),
$$

where $C_{p, p^{\prime}}(t) \equiv \chi \sum_{q, q^{\prime}} \mu_{q}^{\star}(t) \mu_{q^{\prime}}(t) V_{p, p^{\prime}, q, q^{\prime}}$ are defined via the overlap sums $V_{p, p^{\prime}, q, q^{\prime}} \equiv \sum_{n} \phi_{n}^{(p)} \phi_{n}^{\left(p^{\prime}\right)} \phi_{n}^{(q)} \phi_{n}^{\left(q^{\prime}\right)}$. The coefficients $C_{p, p^{\prime}}(t)$ are real and depend on time through the coordinates themselves.

In the limit of small amplitude $\left|\mu_{p}\right|$, cubic terms in eq. (2) are higher order and may be neglected during some time. The linear behavior is $\mu_{p}(t) \approx \mu_{p}(0) e^{-i \omega_{p} t}$. Then during that interval of time, coefficients $C_{p, p^{\prime}}(t)$ are almost periodic functions of time (i.e., with a countable set of periods $), C_{p, p^{\prime}}(t) \approx \chi \sum_{q, q^{\prime}} V_{p, p^{\prime}, q, q^{\prime}} \mu_{q}^{\star}(0) \mu_{q^{\prime}}(0) e^{i\left(\omega_{q}-\omega_{q^{\prime}}\right) t}$.

\footnotetext{
${ }^{1}$ An almost periodic function $f(t)$ can be written as an absolutely convergent series, $f(t)=\sum_{n} f_{n} e^{i \omega_{n} t}$, where the set of frequencies $\omega_{n}$ is countable and $\sum_{n}\left|f_{n}\right|<+\infty$. 17]
}

From eq. (2), $\frac{d}{d t}\left|\mu_{p}\right|^{2}=2 \sum_{p^{\prime} \neq p} C_{p, p^{\prime}}(t) \operatorname{Im}\left(\mu_{p}^{\star} \mu_{p^{\prime}}\right)$, defining the norm current $J_{p^{\prime} \rightarrow p}$ between two different Anderson modes $p \neq p^{\prime}$ as

$$
\begin{array}{r}
J_{p^{\prime} \rightarrow p}=2 C_{p, p^{\prime}}(t) \operatorname{Im}\left(\mu_{p}^{\star} \mu_{p^{\prime}}\right) \\
\approx 2 \chi \sum_{q, q^{\prime}}\left[V_{p, p^{\prime}, q, q^{\prime}}\left|\mu_{q}(0)\right| \cdot\left|\mu_{q^{\prime}}(0)\right| e^{i\left(\left(\omega_{q}-\omega_{q^{\prime}}\right) t-\left(\alpha_{q}-\alpha_{q^{\prime}}\right)\right)}\right] \\
\times\left|\mu_{p}(0)\right| \cdot\left|\mu_{p^{\prime}}(0)\right| \sin \left(\left(\omega_{p}-\omega_{p^{\prime}}\right) t-\left(\alpha_{p}-\alpha_{p^{\prime}}\right)\right),
\end{array}
$$

where $\alpha_{p}$ is the initial phase of $\mu_{p}(0)=\left|\mu_{p}(0)\right| e^{-i \alpha_{p}}$. This current is almost periodic in time, and its oscillations should be small in order that $\left|\mu_{p}(t)\right|^{2}$ remains approximately constant, so that $\mu_{p}(t) \approx \mu_{p}(0) e^{-i \omega_{p} t}$ remains valid for all times. Since time integration of the current yields denominators $\omega_{q}-\omega_{q^{\prime}} \pm\left(\omega_{p}-\omega_{p^{\prime}}\right)$ which may be small, this condition requires that there should be no strong resonances between any pairs of sites $p \neq p^{\prime}$. We consider resonances to be weak enough when the numerator is smaller than the denominator for all terms, i.e.,

$$
\left|\omega_{q}-\omega_{q^{\prime}} \pm\left(\omega_{p}-\omega_{p^{\prime}}\right)\right| \gtrsim \kappa\left|\chi V_{p, p^{\prime}, q, q^{\prime}}\right| \cdot\left|\mu_{q}(0)\right| \cdot\left|\mu_{q^{\prime}}(0)\right|
$$

for all $q$ and $q^{\prime}$, where $\kappa$ is of order 1 (Chirikov criterion). Note also that \pm can be dropped, since the same condition is obtained if $q$ and $q^{\prime}$ are exchanged.

We assume now in order to fix the ideas that $\omega_{p}$ are random numbers distributed in some interval with a smooth probability law with maximum density $P_{0}$. Each resonance $p, p^{\prime}, q, q^{\prime}$ has thus a probability with the upper bound $2 P_{0} \kappa\left|\chi V_{p, p^{\prime}, q, q^{\prime}}\right| \cdot\left|\mu_{q}(0)\right| \cdot\left|\mu_{q^{\prime}}(0)\right|$ to occur. The probability $P_{R}$ that there is at least one resonance in the system for this initial condition, is bounded by the sum of these probability bounds divided by 2 (resonance $p, p^{\prime}, q, q^{\prime}$ is equivalent to $\left.p^{\prime}, p, q^{\prime}, q\right)$ :

$$
P_{R} \leq P_{0} \kappa|\chi| \sum_{q, q^{\prime}}\left|\mu_{q}(0)\right| A_{q, q^{\prime}}\left|\mu_{q^{\prime}}(0)\right|,
$$

where $A_{q, q^{\prime}} \equiv \sum_{p \neq p^{\prime}}\left|V_{p, p^{\prime}, q, q^{\prime}}\right|$. Then we obtain

$$
P_{R} \leq P_{0} \kappa|\chi| \cdot\|\mathbf{A}\| \cdot\left\|\left\{\mu_{q}(0)\right\}\right\|^{2},
$$

with $\mathbf{A}=\left\{A_{q, q^{\prime}}\right\}$ and the norm

$$
\|\mathbf{A}\|=\sup _{\mathbf{X}} \frac{\|\mathbf{A} \cdot \mathbf{X}\|}{\|\mathbf{X}\|} .
$$

$\|\mathbf{A}\|$ may be equivalently defined as the smallest upper bound of the eigenspectrum of $\mathbf{A}$.

The probability to have at least one resonance is thus directly related to the norm square $\left\|\left\{\mu_{q}(0)\right\}\right\|^{2}$ of the initial condition (this was also found numerically in [13]). The probability $P_{N} \equiv 1-P_{R}$ to have no resonance thus has a lower bound, $P_{N} \geq 1-P_{0} \kappa|\chi| \cdot\|\mathbf{A}\| \cdot\left\|\left\{\mu_{q}(0)\right\}\right\|^{2}$, so that when the norm of the initial condition is small enough,

$$
\left\|\left\{\mu_{q}(0)\right\}\right\|^{2}<\frac{1}{P_{0} \kappa|\chi| \cdot\|\mathbf{A}\|},
$$


we obtain $P_{N}>0$. Thus, if we can prove that the norm $\|\mathbf{A}\|$ is not infinite, the probability to have no resonance will be nonvanishing, and go to 1 when the norm of the initial condition goes to zero.

An upper bound for $\|\mathbf{A}\|$ can be obtained from

$$
\begin{array}{r}
\| \mathbf{A}|| \leq \sup _{q} \sum_{q^{\prime}}\left|A_{q, q^{\prime}}\right| \\
\leq \sup _{q} \sum_{p \neq p^{\prime}, q^{\prime}} \sum_{n}\left|\phi_{n}^{(p)}\right| \cdot\left|\phi_{n}^{\left(p^{\prime}\right)}\right| \cdot\left|\phi_{n}^{(q)}\right| \cdot\left|\phi_{n}^{\left(q^{\prime}\right)}\right| \\
<\sup _{q} \sum_{n}\left(\sum_{p}\left|\phi_{n}^{(p)}\right|\right)^{3}\left|\phi_{n}^{(q)}\right| .
\end{array}
$$

If the eigenstates are exponentially localized, then $\sum_{p}\left|\phi_{n}^{(p)}\right|<+\infty$ and $\sum_{n}\left|\phi_{n}^{(q)}\right|<+\infty$. If we assume these are bounded for all $n$ or $q$ by the same constant $S$, then $\|\mathbf{A}\|<S^{4}<+\infty 2$ Note that since $\sum_{n}\left|\phi_{n}^{(q)}\right|^{2}=1$, if the localization length increases and diverges, then $S>\sum_{n}\left|\phi_{n}^{(q)}\right| \rightarrow \infty$. To obtain a reasonable estimate of $\|\mathbf{A}\|$ we assume an exponential bound for all eigenstates,

$$
\left|\phi_{n}^{(p)}\right|<K \sqrt{\frac{1-\lambda^{2}}{1+\lambda^{2}}} \lambda^{|n-p|},
$$

where $K$ is some constant and $\lambda=e^{-1 / \xi}$, where $\xi$ is the localization length. Then $S^{4}=K^{4} \frac{(1+\lambda)^{6}}{\left(1+\lambda^{2}\right)^{2}} \frac{1}{(1-\lambda)^{2}}$. When the localization length diverges at weak disorder we find

$$
\|\mathbf{A}\| \lesssim 16 K^{4} \xi^{2} .
$$

Consequently this upper bound for the norm diverges, suggesting that $\|\mathbf{A}\|$ might also diverge in the same way.

We did not consider the probabilities of resonances at higher orders $6,8, \ldots$ which are cumbersome to calculate. The correction on the bound of $P_{R}$ would be higher order in $\left\|\left\{\mu_{q}(0)\right\}\right\|^{2}$ and thus could be neglected in the limit of small norm. We conjecture that at each order $2 p$, these probabilities can also be bounded by convergent series multiplied with $\left\|\left\{\mu_{q}(0)\right\}\right\|^{2(p-1)}$. The probability of higher order resonance is expected to decay exponentially with the order. Thus when the norm of the initial condition is not too large, we would expect that the probability of no resonance at any order is non-vanishing and still bounded from below, going to 1 as the norm goes to zero.

Note that for finite systems with size $N$, the linear spectrum is always discrete and the series for bounding $P_{R}$ become finite sums, implying $\|\mathbf{A}\|<+\infty$. Then, we

\footnotetext{
${ }^{2}$ If $\mu_{q}(0)$ is not arbitrarily chosen, we may get a better upper bound for the existence of KAM tori. For example, if $\mu_{q}(0)=$ $\delta_{q, q_{0}}\left\|\left\{\mu_{q}(0)\right\}\right\|$ is initially localized at a single Anderson mode $q^{\prime}=q_{0}\left[14\right.$, we have $P_{R} \leq P_{0} \kappa|\chi|\left(\sup _{q^{\prime}}\left|A_{q^{\prime}, q^{\prime}}\right|\right) \cdot\left\|\left\{\mu_{q}(0)\right\}\right\|^{2}$ where $\sup _{q^{\prime}}\left|A_{q^{\prime}, q^{\prime}}\right|<\|\mathbf{A}\|$. Thus, initial wave packets which are close to single Anderson modes survive much better as almost periodic solutions than those which are arbitrarily spread (in Anderson space) at the same norm. This effect is especially important when the localization length is large since then $\sup _{q^{\prime}}\left|A_{q^{\prime}, q^{\prime}}\right|<<\|\mathbf{A}\|$. The same is true if the initial wavepacket is split into several packets with smaller norm which are far apart at the scale of the localization length.
}

know that the conclusion of our empirical argument is consistent with KAM theory, predicting the existence of $N$-dimensional invariant tori of quasiperiodic solutions at small enough amplitude (or equivalently, for finite systems, small enough norm) with a probability going to 1 at zero amplitude. Our conjecture is that this argument also holds for infinite systems provided $\|\mathbf{A}\|<+\infty$. This situation occurs when the linear spectrum is purely discrete with exponentially localized eigenstates, but is not fulfilled when it contains an absolutely continuous part. Then we would conclude, that the norm region for initial conditions where KAM tori may exist shrinks to zero when the localization length diverges, approaching the limit without disorder where the linear spectrum is absolutely continuous and no $l_{2}$ almost periodic exact solution could survive due to radiaton.

Attempting to distinguish numerically KAM tori among other solutions in the random DNLS equation, we first consider that trajectories of KAM tori are almost periodic in time, and use the Harald Bohr theorem:

If a function $f(t)$ is almost periodic, then for any $\varepsilon>0$, there exists a relatively dense set of translations $\tau$ such that $|f(t)-f(t+\tau)|<\varepsilon$ for all $t \in]-\infty,+\infty[$. In other words, there exists a diverging subsequence $\tau_{n}$ fulfilling this property with $\tau_{n}<\tau_{n+1}$ and $\tau_{n+1}-\tau_{n}$ bounded.

Thus, for KAM-like tori, recurrences should be observed numerically in all quantities which depend on time such as local coordinates at arbitrary sites, momenta, participation number, etc. Typically, if recurrences appear for one of these quantities, they are found also for any other. Some problems with this method are obviously that

(i) the Harald Bohr theorem can be checked only for finite times $\tau \in[0, T]$ and $t \in[0, T]$, where $T$ is the time of integration, and for finite-size systems;

(ii) then $\varepsilon$ cannot be chosen too small for avoiding that recurrences become too rare, and thus that the corresponding pseudoperiod exceeds the integration time;

(iii) the system size should be sufficient in order that the amplitude is practically zero at the edge, so that boundary effects can be neglected during the integration time $T$.

(iv) the integration accuracy should be good enough, for avoiding numerical drift from KAM-like tori to neighboring chaotic trajectories. In practice, the relative error in the conserved quantities are kept at the order $10^{-6}$ or smaller in all simulations, with consistency checks for smaller systems reaching accuracies $10^{-8}-10^{-10}$.

If there are KAM tori persisting over infinite time, we should expect that their structure as some model parameter varies is a fat Cantor set with infinitely many gaps due to resonances at all orders, but nonvanishing Lebesgue measure (or nonvanishing probability). Thus, one should find that the probability (in the space of initial conditions) that recurrence persists over a time $T$ does not shrink to zero as $T$ becomes very large. However, high order resonances (corresponding to small gaps) should manifest only after very long integration time. These trajectories may look almost periodic and exhibit recurrence over relatively 

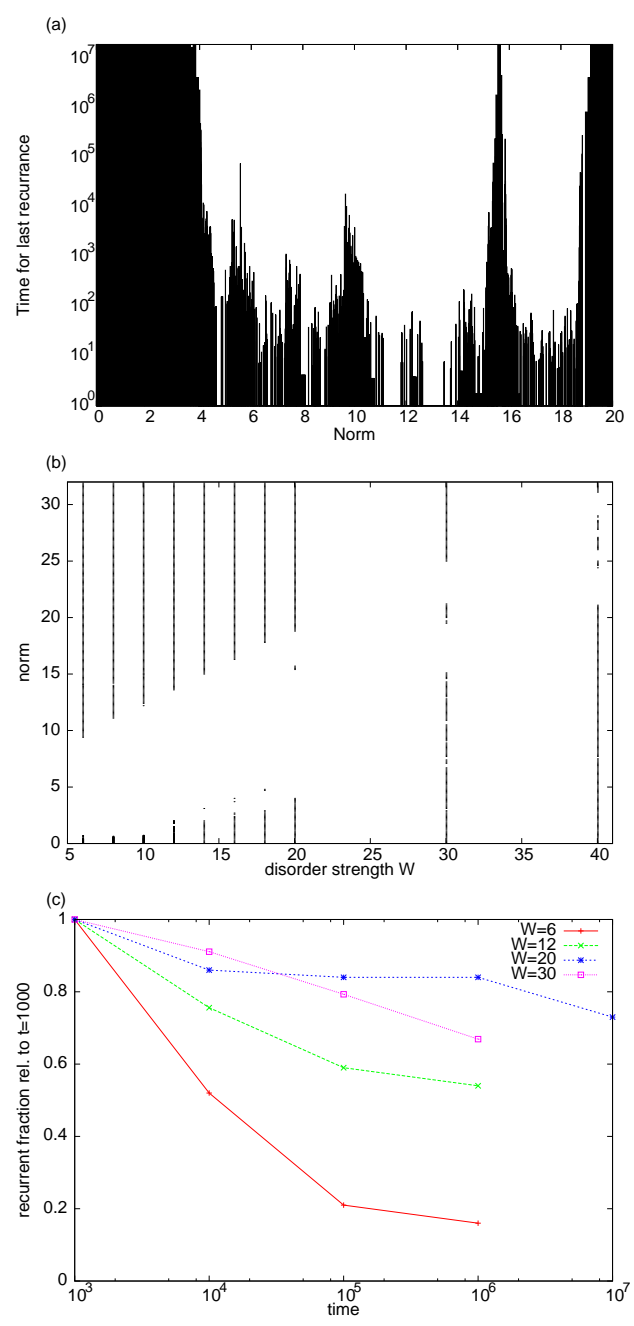

Fig. 1: (a) Last observed time for recurrence $(\varepsilon / \mathcal{N}=0.02)$ in $\left|\psi_{n_{0}}\right|^{2}$ versus norm in a particular disorder realization with $W=20$. (b) Sets of $T$-recurrent trajectories at $T=10^{4}$ for various disorder strengths $W . \varepsilon / \mathcal{N}$ varied from $0.1(W=6)$ to $0.005(W \geq 14)$. (c) Fraction of the trajectories $T$-recurrent at $T=10^{3}$ (excluding the high-norm self-trapped regime) which remain $T$-recurrent also at longer times, for various disorder strengths. At final integration times, $\varepsilon / \mathcal{N}$ is, respectively, 0.03 $(W=6), 0.003(W=12), 0.001(W=30)$, and $0.0005(W=$ 20). In all figures, the disorder realization is the same, the initial condition $\psi_{n}(t=0)=\sqrt{\mathcal{N}} \delta_{n, n_{0}}$ with $\epsilon_{n_{0}} \approx 0.46529 \mathrm{~W}$, $C=1, \chi=-1$, and system size $N=500$.

long times, before they blow up as chaotic trajectories. (Such trajectories were numerically identified in [13] as belonging to a "regime I" of rather small norm and/or strong disorder.) Generally, all trajectories which remain recurrent after a time $T$ will be termed T-recurrent.

An illustration is given in fig. 1(a), showing the last observed recurrence time as a function of norm for a singlesite initial condition in a particular disorder realization for rather strong disorder $W=20$ (we chose the initial-site energy $\epsilon_{n_{0}}$ rather close to the upper band edge, in order that an increasing negative nonlinearity will "scan" most possible resonances inside the band). Any horizontal intersection of this graph at a given time $T$ yields the set of initial norm generating $T$-recurrent trajectories. As can be seen, many trajectories remain recurrent for times larger than $10^{7}$. In addition to the regime of small norm (here $\mathcal{N} \lesssim 3.6$ ) expected from our argument above, there is also a regime of recurrent states for $\mathcal{N} \gtrsim 19$, as well as a small interval around $\mathcal{N} \approx 15.6$. The recurrent trajectories in the large-norm regime are related to the fact that, above some threshold norm, an increasingly larger part of the norm will self-trap around the initial site $n_{0}[12$. The total norm available for the rest of the lattice will then actually decrease, and essentially the same argument as above could be used in support of a KAM-like regime. Note also that the simultaneous limit of strong disorder and large norm, $W \rightarrow \infty, \mathcal{N} \rightarrow \infty$ with $\chi \mathcal{N} / W$ finite, is equivalent (rescaling time) to the anticontinuous (uncoupled) limit $C=0$ which is also integrable, and where the linear localization length is vanishing. In this limit, for any initial condition, each anharmonic oscillator $n$ exhibits a periodic motion with frequency $\omega_{n}^{\prime} \neq \epsilon_{n}$ different from the corresponding onsite linear frequency. We may thus expect a KAM-like regime also close to this limit.

Thus, though numerics cannot provide a rigorous proof, the most plausible interpretation is that that there is an underlying Cantor set of initial conditions generating KAM tori, persisting over infinite time and infinitely large systems. Note also that the gap structure of Fig. 1(a) is reminiscent of the "stickiness" phenomenon in lowdimensional systems, where many initial conditions close to (but outside) the Cantor set of KAM tori remain close for long times before they finally escape (compare, e.g., with Figs. 7 and 10 in 18 for the standard map).

The variation of the sets of $T$-recurrent trajectories with the disorder strength is illustrated by fig. 1(b), for a rather modest time $T=10^{4}$. As predicted, there is always a small-norm regime where most trajectories are $T$-recurrent, the size of which grows with increasing disorder strength. There is also always a high-norm $T$-recurrent regime with lower boundary increasing with increasing disorder, since the norm necessary for efficient self-trapping increases linearly with $W$ for a single-site initial condition 12. In-between these two regimes, for larger $W$ there are also several intermediate regimes of $T$-recurrence, separated by gaps with non-recurrent (chaotic) trajectories. For smaller $W$ the relative sizes of these gaps grow, and they merge into one single main gap of trajectories which typically show a chaotic time-evolution and spread subdiffusively [9 11,13] (for long but possibly finite times).

Similar pictures as fig. 1(b) can be obtained for larger times, although obtaining a good resolution with sufficient numerical accuracy to clearly identify recurrences makes it very time-consuming for times larger than $\sim 10^{6}$. As a rule of thumb, to determine persistent recurrences $\varepsilon$ is divided by two for each order of magnitude in time. In fig. 1(c), we give an example showing how the measure of the set of $T$-recurrent initial conditions varies with $T$, for differ- 
ent strengths $W$ of the same disorder realization. In order to obtain a finite set, we here exclude recurrent trajectories belonging to the high-norm (self-trapped) regime, and moreover for comparison we normalize the sets by dividing with the number of $T$-recurrent trajectories at $T=10^{3}$. The data of fig. 1(c) suggest the existence of an asymptotic set with a nonvanishing measure at infinite time. However, for stronger disorder, it is clear from fig. 1(c) that to get a clear picture of the asymptotic measure of this set, considerably longer integration times would be needed. This is due to the fact that the fraction of "sticky" trajectories, which are $T$-recurrent over very large but finite times, increases with the disorder strength (cf. fig. 1(a)).

Thus, the numerical study of $T$-recurrent trajectories for finite times can give us only an indication about the true nature of the KAM-like trajectories, and in particular the presence of long-time "sticky" trajectories makes it extremely difficult, e.g., to resolve the Cantor-set structure of resonances in the low-norm regime within a reasonable amount of computer time. We therefore now turn to discusss another technique to numerically distinguish KAM tori, in terms of the tangent map and the corresponding finite-time Lyapunov exponents. Small perturbations of eq. (1) yield the Hill equation

$$
i \dot{\eta}_{n}=\left(\epsilon_{n}+2 \chi\left|\psi_{n}\right|^{2}\right) \eta_{n}+\chi \psi_{n}^{2} \eta_{n}^{\star}-C\left(\eta_{n+1}+\eta_{n-1}\right) .
$$

If eq. (11) possesses an almost periodic solution with the discrete set of frequencies $\omega_{1}, \omega_{2}, \ldots, \omega_{p}, \ldots$, corresponding to KAM tori with full dimension,

$\psi_{n}=F_{n}\left(\omega_{1} t+\alpha_{1}, \omega_{2} t+\alpha_{2}, \ldots, \omega_{p} t+\alpha_{p}, \ldots ; \omega_{1}, \omega_{2}, \ldots, \omega_{p}, \ldots\right)$,

where $F_{n}\left(x_{1}, x_{2}, \ldots x_{p}, \ldots ; \omega_{1}, \omega_{2}, \ldots, \omega_{p}, \ldots\right)$ is $2 \pi$-periodic with respect to $x_{1}, x_{2}, \ldots, x_{p}, \ldots$, then $\eta_{n}(t)=\frac{\partial F_{n}}{\partial x_{p}}$ is an almost periodic solution of eq. (5). One gets a complete basis of solutions of eq. (5) by adding also the solutions $\eta_{n}(t)=\frac{d F_{n}}{d \omega_{p}}=t \frac{\partial F_{n}}{\partial x_{p}}+\frac{\partial F_{n}}{\partial \omega_{p}}$, where also $\frac{\partial F_{n}}{\partial \omega_{p}}$ is almost periodic. Consequently, if the solution of eq. (11) corresponds to a KAM torus, the general solution of eq. (55) grows linearly as a function of time. Note also that for any solution $\psi_{n}$ to eq. (11), there is a trivial solution $\eta_{n}=i \psi_{n}$ to eq. (5) corresponding to a global phase rotation. Numerically, we remove this component by subtracting the projection of $\eta_{n}(t)$, obtained by integrating a randomized initial condition $\eta_{n}(0)$, on this vector.

Thus, considering the total norm of the perturbation divided by $t, \frac{1}{t}\|\eta(t)\|$ where $\|\eta\|=\sqrt{\sum_{n}\left|\eta_{n}\right|^{2}}$, this quantity will exhibit bounded oscillations for all times if $\psi_{n}$ corrsponds to a KAM torus, and diverge exponentially with a positive Lyapunov exponent for any chaotic trajectory. A numerical illustration is given in fig. 22(a), showing a narrow resonance gap in the low-amplitude KAM-like regime around $\mathcal{N}=0.600$ for disorder strength $W=12$.

From the so obtained numerical solutions $\eta_{n}(t)$, we calculate finite-time Lyapunov exponents as $\Lambda\left(t_{M}\right)=$ $\frac{1}{t_{M}} \sum_{m=1}^{M} \log \frac{\left\|\eta\left(t_{m}\right)\right\|}{\left\|\eta\left(t_{m}-1\right)\right\|}$, where $t_{0}=0$, and $t_{m}$ are chosen
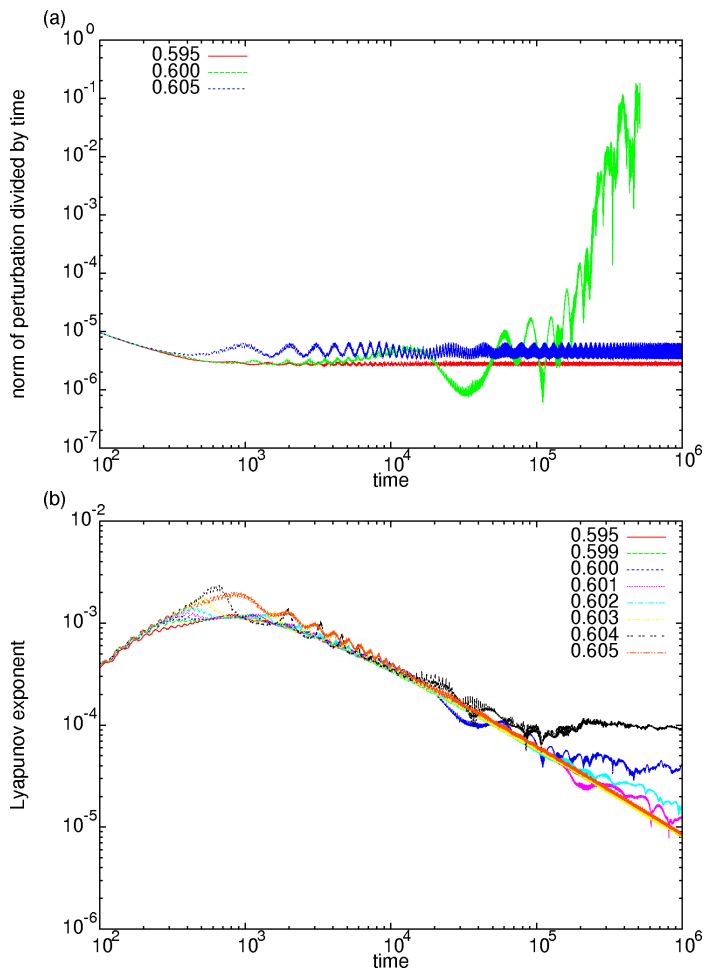

Fig. 2: (a) Total norm of perturbation $\eta_{n}(t)$ divided by time, for three solutions corresponding to single-site initial conditions $\psi_{n}$ with slightly different $\mathcal{N} \approx 0.6$. (b) Finite-time Lyapunov exponents for 8 solutions in the same regime as in (a). At time $10^{6}$, the upper curves in (b) correspond, from top to bottom, to $\mathcal{N}=0.604,0.600,0.602,0.601$, while the lower curves for $\mathcal{N}=0.595,0.599,0.603,0.605$ all follow very closely a curve $\sim$ $\log t / t$, as expected for KAM tori. Disorder strength $W=12$, other parameters and disorder realization same as in fig. 1

to correspond to a Poincaré section defined by $\left|\psi_{n_{0}}(t)\right|^{2}$ having a local maximum at each $t=t_{m}$. Thus, for a recurrent trajectory, the optimal recurrence times $\tau_{k}$ form a subset of the $t_{m}$. Moreover, for an almost-periodic trajectory with a time-linear growth of $\|\eta\|, \Lambda(t)$ should decrease asymptotically to zero as $\Lambda(t) \sim \log (t) / t$ for large times. As can be seen from the example in fig. 2(b), the behaviour of $\Lambda(t)$ in the neighborhood of sharp resonances is very sensitive to small parameter variations. In this example, the trajectories are seen to be (possibly weakly) chaotic in the interval $0.600 \leq \mathcal{N} \leq 0.602$ and in an even narrower interval around $\mathcal{N} \approx 0.604$, while apparently almost-periodic trajectories (with no visible deviations from the asymptotic behaviour $\Lambda(t) \sim \log (t) / t$ for times larger than $10^{6}$ ) are seen e.g. for $\mathcal{N}=0.595,0.599,0.603$ and 0.605 . Thus, this again supports the existence of a finite-measure Cantor set of almost-periodic KAM tori.

Comparing the numerical results from the analysis of recurrences and tangent map, they are consistent in the sense that when recurrences are lost, there is a clear deviation in $\|\eta\|$ from time-linear growth, and in most cases the growth is exponential with well-defined non-zero $\Lambda(t)$ (at 


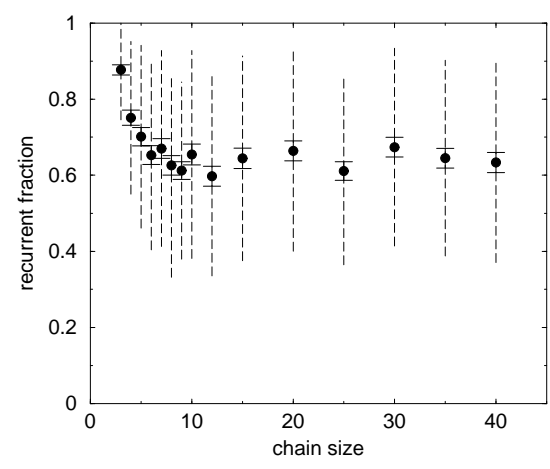

Fig. 3: Ensemble-averaged fractions of trajectories $T$-recurrent at $T=2 \cdot 10^{5}$ versus chain size, for random single-site initial conditions with $0<\mathcal{N} \leq 20$ and disorder strength $W=12$. 100 different realizations were used for each size, and dashed lines show the standard deviations of the distributions. Trajectories were considered $T$-recurrent if $\Lambda(T)<10^{-4}$ (cf. fig. 21).

least for long times). However, generally (and in particular for strong disorder with many "sticky" trajectories), the tangent-map criterion is considerably more sensitive, and may signal a chaotic behaviour several orders of magnitude in time before recurrence is finally lost.

Generally, we find the KAM-like trajectories to be exponentially localized, decaying essentially with the largest linear localization length $\xi\left(e . g, \xi_{\max } \approx 1.1\right.$ for $W=12$ as in fig. 21). Thus we may expect that, for stronger disorder, the KAM-like trajectories observed for large systems should be only slight perturbations of true KAM tori existing for short chains. This was also confirmed numerically. An illustration is given in fig. [3 where, for $W=12$ and single-site initial conditions $\psi_{n}(0)=\sqrt{\mathcal{N}} \delta_{n, n_{0}}$ with $0<\mathcal{N} \leq 20$, we calculate the fraction of the total number of trajectories remaining $T$-recurrent after $T=2 \cdot 10^{5}$, for chain lengths with $3 \leq N \leq 40$. For each chain length, we used 100 different (independent) disorder realizations, and determined ensemble averages and standard deviations (note that for small chains, ensemble statistics can be done with reasonable computational effort). As can be seen, already for $N=6\left(\approx 5 \xi_{\max }\right)$ this fraction has essentially converged to its large-chain limit. The large spread between different realizations is essentially due to the fact that $\epsilon_{n_{0}}$ also is chosen randomly: if $\epsilon_{n_{0}}$ is close to the linear band bottom, self-trapping occurs already for small values of $\mathcal{N}$, and therefore most trajectories will be KAMlike. By contrast, realizations with $\epsilon_{n_{0}}$ close to the band top as in figs. 1 2 should yield the largest amount of resonances. Indeed, for the particular realization used for the large-size simulations in figs. 1,2, we obtain for $W=12$ a total fraction of $38 \% T$-recurrent trajectories in the interval $0<\mathcal{N} \leq 20$ after $T=10^{5}$ (decreasing to $37 \%$ at $T=10^{6}$ ), essentially coinciding with the lower bounds in fig. 3 (i.e., this realization does not behave exceptionally).

In summary, our empirical and numerical arguments suggest that in the infinite random DNLS model (1), there are two kinds of initial wavepackets both occuring with nonzero probability: (i) those generating spatially localized (non-spreading), almost periodic solutions (KAM tori); and (ii) wave-packets which are initially chaotic and spreading [9 11, 13]. Our results should stimulate further attempts towards more rigorous treatments, as well as more detailed numerical studies of the role of such tori in various situations. Ongoing work suggests, e.g., that also large-norm wavepackets extended on many sites could generate KAM tori with high probability if the norm density is small enough. Thus, the spreading of chaotic wavepackets may after long times drastically slow down, if they become sticky to such tori. The KAM-like trajectories may also be experimentally observable, e.g., in disordered waveguide arrays [15], or in disordered bosonic systems [19].

$$
* * *
$$

We thank S. Flach, D. Krimer, Ch. Skokos and J. Bodyfelt for useful suggestions. MJ acknowledges support from the Swedish Research Council.

\section{REFERENCES}

[1] For a simple presentation, see, e.g., Gignoux F. and Silvestre-Brac, B., Solved Problems in Lagrangian and Hamiltonian Mechanics (Springer, Dordrecht) 2009.

[2] Fröhlich J., Spencer T. and Wayne C. E., J. Stat. Phys., 42 (1986) 247.

[3] Pöschel J., Commun. Math. Phys., 127 (1990) 351.

[4] Froeschlé C. and Scheidecker J.-P., Phys. Rev. A, 12 (1975) 2137; Froeschlé C., ibid., 18 (1978) 277.

[5] Aubry S. and Schilling R., Physica D, 238 (2009) 2045.

[6] Sievers A. J. and Takeno S., Phys. Rev. Lett., 61 (1988) 970; MacKay R. S. and Aubry S., Nonlinearity, 7 (1994) 1623; Flach S. and Gorbach A., Phys. Rep., 467 (2008) 1.

[7] Albanese C. and Fröhlich J., Commun. Math. Phys., 138 (1991) 193.

[8] Kopidakis G. and Aubry S., Phys. Rev. Lett., 84 (2000) 3236; Physica D, 130 (1999) 155; 139 (2000) 247.

[9] Shepelyansky D. L., Phys. Rev. Lett., 70 (1993) 1787.

[10] Molina M. I., Phys. Rev. B, 58 (1998) 12547.

[11] Pikovsky A. S. and Shepelyansky D. L., Phys. Rev. Lett., 100 (2008) 094101.

[12] Kopidakis G. et al., Phys. Rev. Lett., 100 (2008) 084103.

[13] Flach S, Krimer D. O. and Skokos Ch., Phys. Rev. Lett., 102 (2009) 024101; 102 (2009) 209903; Skokos CH. et al., Phys. Rev. E, 79 (2009) 056211.

[14] Fishman S., Krivolapov Y. and Soffer A, Nonlinearity, 22 (2009) 2861.

[15] Lahini Y. et al., Phys. Rev. Lett., 100 (2008) 013906.

[16] Bourgain J. and Wang W.-M., J. Eur. Math. Soc., 10 (2008) 1.

[17] Bredikhina E. A., Encyclopaedia of Mathematics, edited by Hazewinkel M. (Springer, Berlin) 2002, sect. Almostperiodic function.

[18] Contopoulos G. et al., Cel. Mech. Dyn. Astron., 67 (1997) 293. 
[19] Deissler B. et al., Nature Physics, 6 (2010) 354. 